\title{
Beach Handball and Beach Volleyball as Means Leading to Increasing Physical Activity of Recreational Sportspeople-Pilot Study
}

\author{
Jan Bělka ${ }^{1}$, Karel Hůlka ${ }^{1}$, Michal Šafár ${ }^{2}$, Radim Weisser ${ }^{1}$ and Julie Chadimova ${ }^{1}$ \\ 1. Department of Sport, Faculty of Physical Culture, Palacky University, Olomouc 77900, Czech Republic \\ 2. Department of Natural Sciences in Kinanthropology, Faculty of Physical Culture, Palacky University, Olomouc 77900, Czech \\ Republic
}

\begin{abstract}
The sample consisted of sixteen man of aged average 24.5 year, average height $181.9 \mathrm{~cm}$ and weight $80.4 \mathrm{~kg}$. The average of static pulse rate was 56.4 beats $\min ^{-1}$. The average heart rate max was $189.7 \pm 7.8$ beats $\min ^{-1}$. For the calculation of $\mathrm{HR}_{\max }(\mathrm{maximal}$ heart rate), a mathematical figure $\mathrm{HR}_{\max }=207-(0.7 \times$ age $)$ was used. Probands have participated on the same day on a tournament in beach and handball tournament. HR (heart rate) was monitored via Sport Tester Polar. The results were evaluated by Polar software. The data was statistically compiled with the use of arithmetic average and standard deviation. The players played three matches in beach volleyball on one set to 21 points (necessary difference of 2 points). One set lasted approximate 13.5 min. After a break, they

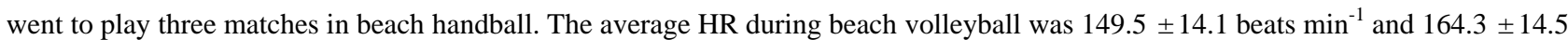

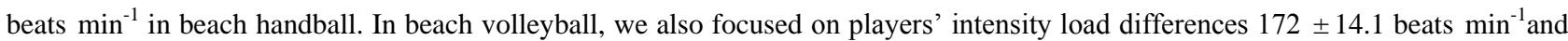
goalkeepers $156.5 \pm 16.6$ beats $\mathrm{min}^{-1}$. In the beach volleyball, players spend most of their playing time on the playing area in the zone intensity load $80 \%-90 \% \mathrm{HR}_{\max }(36 \%$ playing time). In the beach handball, players spend most of their playing time on the playing area in the zone intensity load $90 \%-100 \% \mathrm{HR}_{\max }$ (39\% playing time).
\end{abstract}

Key words: Sport games, heart rate, physical activity, health.

\section{Introduction}

Man as a human being is both morphologically and functionally adapted to the way of life of being able to deal with a difficult physical activity which has always been a part of basic attribution of living and surviving. Health problems and diseases such as obesity, diabetes mellitus II type, hypertension, metabolic cardiovascular syndrome, ischaemic heart disease, peripheral vascular disease, lower back pain, osteoporosis and fractures are mostly connected with the inactive way of living and sedentary jobs, and they are said to be the cause of the mentioned chronic health diseases. The intensified physical activity has a beneficiary potential on better health state by evocating

Corresponding author: Jan Bělka, Ph.D., assistant of professor, research field: sport training, sport condition, team handball and small sided games. E-mail: jan.belka@upol.cz. adaptive changes in most of the organs and systems, their function and regulation in the human body.

One of the most important things for physical education is the human biological need for a physical activity. Satisfying such a need comes with selection. From the natural need for an activity is, as a consequence, a new social need (a motive for social contact) has developed. The actual needs related to physical activity are based on stimulatory tendencies of motivational cluster such as need for change, need for activity repetition, health care need, etc. The necessary condition for doing a sport is the joyful experience. Presently, the joyful experience coming through a physical activity is one of the most important motives of it. We also speak here about an attractiveness of sport in the means of offering a wide range of unconventional, funny and socializing physical 
activities such as co-educated forms of PA (physical activity), meeting with friends, etc. Beach games have become a big temptation not only for professional athletes, but also for common recreational sportspeople.

The basic point of beach games was the active way of doing leisure time activities by the sea or on the beach. The main idea was to relax, have fun, meet friends, gain experience, and have better health condition.

Beach volleyball combines all positive aspects of team sports-ability to play theball, speed and accuracy of movement, flexibility, deemed necessary to link individual actions and thinking of the teammates, etc. To these positive aspects, we can add multilateral influence of the natural factors in outdoor sports [1]. The popularity of beach volleyball is increasing, but it is a sport in the early stages of its development and it is necessary to introduce quality materials to support the development of players and coaches through practical advice [2].

Beach handball is played in a $15 \times 12 \mathrm{~m}$ sandfield. Each equipment has one goalkeeper and three players of field that compete during two halves of 10 minutes each $(2 \times 10 \mathrm{~min})$. They follow the applicable rules of in door handball, with the exception of the zone of change, which is an entire band for each team, and the score of the goalkeepers, which counts double [3].

There are few authors dealing with topics such as basketball, football, volleyball, beach football, indoor football, rugby and who also deals with heart rate monitoring during competition games [4-14]. All of them by Cobos include a recording of heart rates by telemetry, a valid system of measurement [15-17]. It gives us information about heart response when performing exercises that express a huge variability of intensity [18] and, at the same time, it gives us an energetice information about the performed exercise [19].

There are only few surveys done on this topic in beach volleyball [20, 21], beach football [7] and beachhandball [22]. As a consequence to these facts, we have done a research based on load intensity of recreational players in beach volleyball and beach handball.

Research of this study was a part of students' granted competition project nr. 43510007: “Analysis of players' intensity during competitions in sport games (basketball, football, handball and volleyball)" at Palacky University in Olomouc.

The main goal of this research was to compare the intensity load of recreational players in beach volleyball and beach handball in competitions on the basis of analysing heart rate monitoring and to analyse the intensity load of recreational players in beach volleyball and handball competition.

\section{Methods}

\subsection{Participants}

Participants were randomly chosen from students studying physical education and sport in Faculty of Physical Culture on Palacky Univerzity Olomouc, Czech Republic. The research battery consisted of 16 men in the age average of $24.5 \pm 3.74$ years, of average height $181.9 \pm 6.02 \mathrm{~cm}$ and weight $80.4 \pm 7.5 \mathrm{~kg}$. The players' average $\mathrm{HR}_{\text {rest }}$ was $56.4 \pm 6.89$ beats $\mathrm{min}^{-1}$. The average $\mathrm{HR}_{\max }$ was $189.7 \pm 7.8$ beats $\mathrm{min}^{-1}$.

Due to lack of financial and organizational support, it was impossible to make exercise tests for getting the $\mathrm{HR}_{\text {max }}$. As a matter of fact the $\mathrm{HR}_{\max }$ was figured out from this formula: $\mathrm{HR}_{\max }=207-(0.7 \times$ age $)$. The heart rate monitoring was realized by the help of Team Polar monitors. The results were evaluated by Polar software.

\subsection{Data Gathering Description}

Posters informing about the participation on the tournament were posted in advanced. The poster informed about registration to this tournament for men's pairs in beach volleyball and a drawing lot tournament in beach handball. There was an informative meeting for all of the teams about the 
tournament system, rules for both sports and about heart rate monitoring. The monitoring was held in TJ Milo beach courts in Olomouc. The tournament in volleyball was held on four beach courts, and the handball tournament was held on just one court.

The beach volleyball tournament lasted from 9 to 11 o'clock and the drawing lot tournament of three teams (two five-member teams and one six-member team) lasted from 12 to 14 o'clock. Before beach volleyball beginning, all of the probands received the heart rate monitors which they put on their chests. The teams received points from both tournaments to the final list. The weather was sunny and windless; the temperature was about $20^{\circ} \mathrm{C}$.

Each team/pair played three matches for one set to 21 points (two-point difference necessary). Then each proband played two matches in beach handball.

\subsection{Statistical Analysis}

The data was with Software Statistica (9.0 version, StatSoft, Inc., Tulsa, USA) processed by the arithmetical average, percentage formulas, standard deviation and statistical importance-Man-Whitney test. There was a statistical significance $P<0.05$. The statistical calculations were based on the time data received from sport testers (how many seconds the players spent in each load intensity zone). All the probands participated on beach volleyball and handball tournament held in the same day.

\section{Results}

The players played in the beach volleyball tournament three matches each for one set only (end by receiving 21 points, but two-point difference necessary). Each set lasted approx. $13.5 \pm 5.3$ minutes. The players' average HR was $149.5 \pm 14.1$ beats $\min ^{-1}$.There is no significant difference between each load intensity zones (Fig. 1) on the significance level $P$ $<0.05$. The probands mostly, more than 50\%, respectively, appeared in the intensity below an anaerobic threshold $\left(<85 \% \mathrm{HR}_{\max }\right)$. The motion on the court was also influenced by the level of players' skills.

After beach volleyball tournament (sixty-minute break), the probands played tournament (three matches) in beach handball. The average heart rate players was $164.3 \pm 14.5$ beats $\min ^{-1}$ in matches beach handball. The significant difference was while comparing load intensity of in the load intensity zone 90\%-100\% $\mathrm{HR}_{\max }$ and $70 \%-80 \% \mathrm{HR}_{\max }(P=0.0201)$ resp. $<70 \%$ $\mathrm{HR}_{\max }(P=0.0455)$. The matches were interrupted only minimally (imprecise ball passing, shooting out of the goal, fouls etc.) and the level of the matches was appropriate to recreational players. The load intensity was more than $50 \%$ above the anaerobic threshold

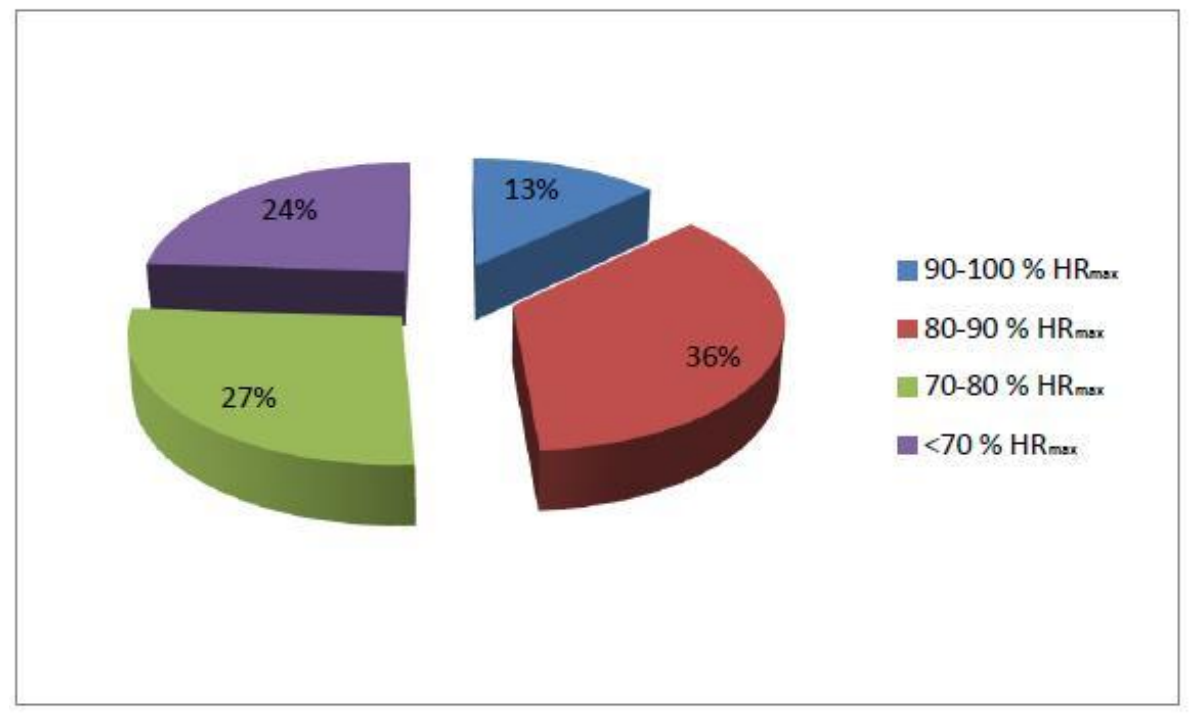

Fig. 1 Load intensity of recreational players in beach volleyball matches. 
(> $85 \% \mathrm{HR}_{\max }$ ). Even the load intensity of goalkeepers was compared here. Due to the tactic and rules specification, there was no statistically significant difference between field players and goalkeepers. It was due to the same game participation of goalkeepers. When losing a ball, the goalkeeper had to leave the court immediately, so the next goalkeeper could run into the goal area. Each team had two goalkeepers.

The only one significant difference was while comparing load intensity of beach handball and beach volleyball matches only in the load intensity zone $90-100 \% \mathrm{HR}_{\max }(P=0.0365)$. We consider higher load intensity to be in the beach handball recreational players. Playing beach handball should lead to improving one's physical ability and fitness factor. Playing beach volleyball should result in weight reduction. This fact is not in comparison with beach football significantly important, but from the practical point of view, it tells us about higher load intensity in beach handball matches (Fig. 3).

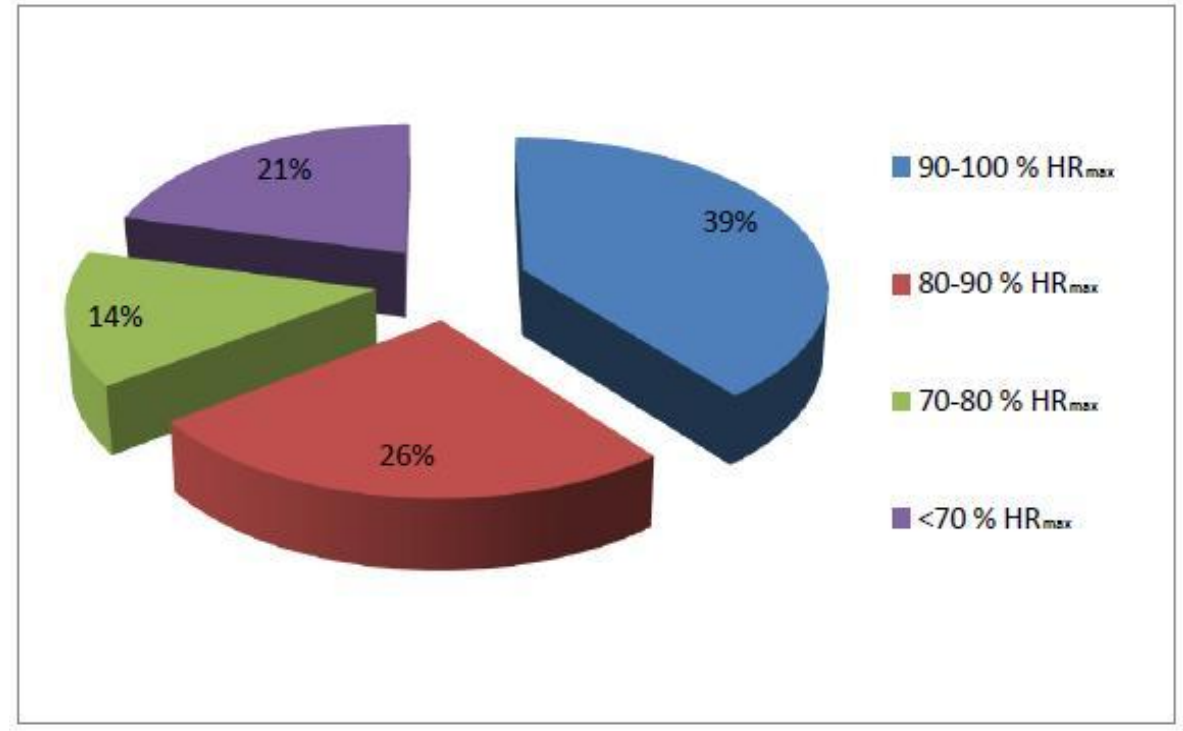

Fig. 2 Load intensity of recreational players in beach handball matches.

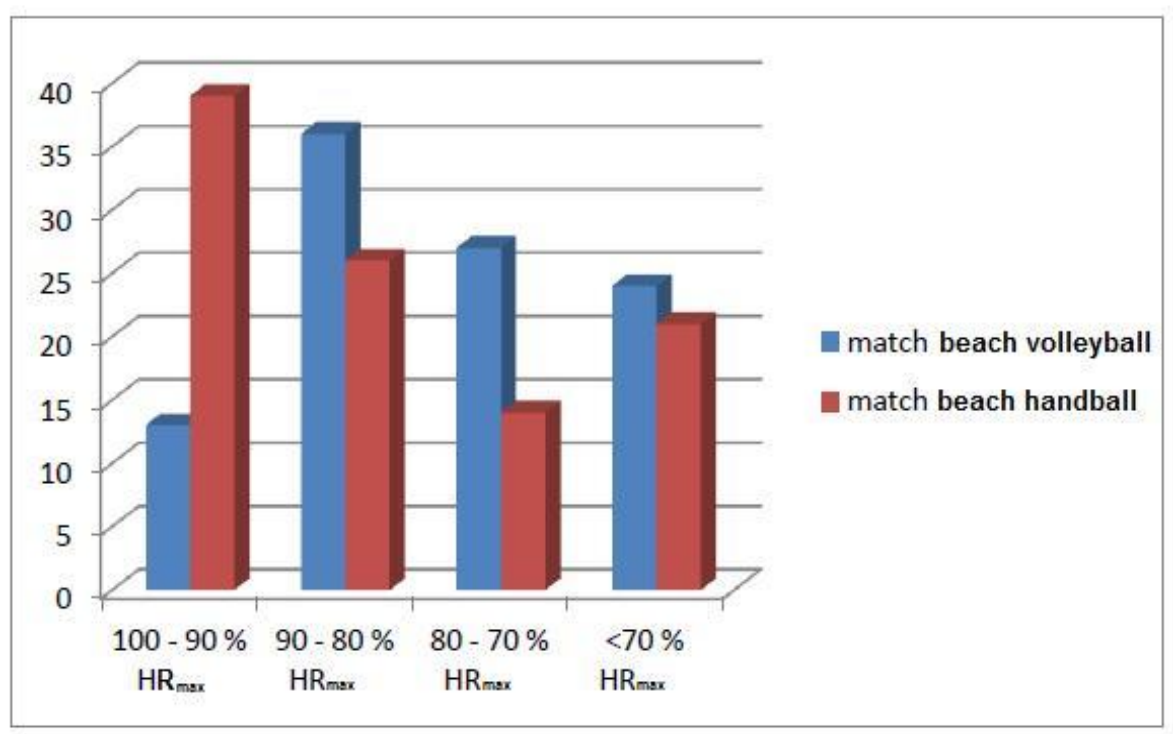

Fig. 3 Comparison of recreational players' intensity in beach handball matches and beach volleyball matches. 


\section{Summary}

The players spent $24 \pm 20.9 \%$ playing time in load low intensity zone $\left(<70 \% \mathrm{HR}_{\max }\right)$ in match beach volleyball. Players beach volleyball spent most playing time $(36 \%)$ in load intensity $80 \%-90 \% \mathrm{HR}_{\max }$. In the match beach handball, players spent most playing time (39\%) in load intensity $90 \%-100 \% \mathrm{HR}_{\max }$ and little playing time in load intensity $70 \%-80 \% \mathrm{HR}_{\max }$.

In beach handball, we monitored the average HR of the players that was $172 \pm 14.1$ beats $\min ^{-1}$ and of the goalkeepers it was $156.5 \pm 16.6$ beats $\mathrm{min}^{-1}$. The players' average HR in volleyball match was $149.5 \pm$ 14.1 beats $\min ^{-1}$.Statistically important difference between each zone was only in the beach handball matches. In comparison with both sports, the statistically important difference was not confirmed. As a matter of fact, only the practical importance can be evaluated. Beach handball recreational players spent in the zone above anaerobic threshold more than $50 \%$ of the whole time in comparison with beach volleyball players, who spent there only $39 \%$.

\section{References}

[1] Kuchukov, V., and Antonov, I. 2004. Beachvolleyball-Management. Sofia: HCA-nPEC.

[2] Jones, H., and Dalanhese, D. 2011. Beach Volleyball Drillbook. Lausanne: Federationinternational de Volleyball.

[3] European Handball Federation. 2005. Beachhandball Rules of the Game. Paper presented at the EHF Course n4 for EHF Beach Handball Referee Candidates, Balatonboglar.

[4] Barbero, J. C., Soto, V. M., Barbero, V., and Granda, J. 2008. "Match Analysis and Heart Rate of Futsal Players during Competition." Journal of Sports Sciences 26 (1): 63-73.

[5] Beam, W. C., and Merill, T. L. 1994. "Analysis of Heart Rates during Female Collegiate Basketball." Medicine and Science in Sports and Exersice 26 (3): 66.

[6] Capranica, L., Tessitore, A., Guidetti, L., and Figura, F. 2001. "Heart Rate and Match Analysis in pre-Pubescent Soccer Players." Journal of Sports Sciences 19 (6): 379-84.

[7] Castellano, J., and Casamichana, D. 2010. "Heart Rate and Motion Analysis by GPS in Beach Soccer." Journal of
Sports Science and Medicine 9 (1): 98-103.

[8] Cohen, M. 1980. "Contribution to the Physiological Study of Basketball.” Ph.D. thesis, Faculte Xavier Bichat, París.

[9] Janeira, M. A., and Maia, J. 1998. "Game Intensity in Basketball. An Interactionist View Linking Time-Motion Analysis, Lactate Concentration and Heart Rate." Coaching \& Sport Science 3 (2): 26-30.

[10] Matthew, D., and Delextrat, A. 2009. "Heart Rate, Blood Lactate Concentration, and Time-Motion Analysis of Female Basketball Players during Competition." Journal of Sports Sciences 27 (8): 813-21.

[11] Mc Innes, S. E., Carlson, J. S, Jones, C. J., and McKenna, M. J. 1995. "The Physiological Load Imposed on Basketball Players during Competition." Journal of Sports Sciences 13 (5): 89-93.

[12] Ramsey, J. D., Ayoub, M. M., Dudek, R. A., and Edgae, H. S. 1970. "Heart Rate Recovery during a Collegue Basketball Game." Journal of Human Sport and Exercise 41 (4): 528-35.

[13] Rodriguez-Alonso, M., Fernández-Garcia, B., Pérezlanaluce, N., and Terrados, N. 2003. "Blood Lactate and Heart Rate during National and International Women's Basketball." The Journal of Sports Medicine and Physical Fitness 43 (4): 432-6.

[14] Vaquera, A. 2008. "Heart Rate Response to Game-Play in Professional Basketball Players." Journal of Human Sport and Exercise 3 (1): 1-9.

[15] Laukkanen, R. M. T., and Virtanen, P. K. 1998. "Heart Rate Monitors: State of the Art." Journal of Sports Sciences 16 (1): 3-7.

[16] Noakes, T. D., Lambert, M. I., and Gleeson, M. 1998. "Heartrate Monitoring and Exercise: Challenges for the Future.” Journal of Sports Sciences 16 (1): 105-6.

[17] Terbizan, D. J., Dolezal, B. A., and Albano, C. 2002. "Validity of Seven Commercially Available Heart Rate Monitors." Measurement in Physical Education \& Exercise Science 6 (4): 243-7.

[18] Moore, A. D., Jr., Lee, S. M., Greenisen, M. C., and Bishop, P. 1997. "Validity of a Heart Rate Monitor during Work in the Laboratory and on the Space Shuttle." American Industrial Hygiene Association Journal 58 (4): 299-301.

[19] Fudge, B. W., Wilson, J., Easton, C., Irwin, L., Clark, J., and Haddow, O. 2007. "Estimation of Oxygen Uptake during Fast Running Using Accelerometry and Heart Rate." Medicine and Science in Sports and Exercise 39 (1): 192-8.

[20] Lorenz, R., Roll, C., Wiebke, D., and Jeschke, D. 2001. "Cardiac and Metabolic Stress during the Beach Volleyball.” Deutsche Zeitschrift für Sportmedizin 52 (2): 7-8.

[21] Michalec, T. 2008. "Analyses of the Load Intenzity 
Players during the of Beach Voleyball Match.” Master thesis, Palacky Univerzity, Faculty of Physical Culture, Olomouc.

[22] Karras, D., Chryssanthopoulous, C., and Diafas, V. 2007.
"Body Fluid Loss during Four Consecutive Beach Handball Matches in High Humidity and Environmental Temperatures." Serbian Journal of Sport Sciences 1 (1): 8-13. 